\title{
Performance of a Real-time Multipurpose 2-Dimensional Clustering Algorithm Developed for the ATLAS Experiment
}

\author{
S. Gkaitatzis, C.-L. Sotiropoulou, Member, IEEE, A. Annovi, K. Kordas \\ on behalf of the ATLAS Collaboration
}

Abstract-In this paper the performance of the 2D pixel
2 clustering algorithm developed for the Input Mezzanine card
3 of the ATLAS Fast TracKer system is presented. Fast TracKer
4 is an approved ATLAS upgrade that has the goal to provide
5 a complete list of tracks to the ATLAS High Level Trigger for
6 each level-1 accepted event, at up to $100 \mathrm{kHz}$ event rate with a
7 very small latency, in the order of $100 \mu \mathrm{s}$. The Input Mezzanine
8 card is the input stage of the Fast TracKer system. Its role is
9 to receive data from the silicon detector and perform real time
10 clustering, thus to reduce the amount of data propagated to the
11 subsequent processing levels with minimal information loss. We
12 focus on the most challenging component on the Input Mezzanine
13 card, the $2 D$ clustering algorithm executed on the pixel data. We
14 compare two different implementations of the algorithm. The
15 first is one called the ideal one which searches clusters of pixels
16 in the whole silicon module at once and calculates the cluster
17 centroids exploiting the whole available information, included
18 the precise sharing of charge produced by the particle between
19 contiguous pixels of the cluster. The second one uses a sliding
20 window technique to identify clusters of contiguous pixels, one at
21 a time. In addition, a simplified centre of mass is calculated as the
22 center of a bounding box which contains the cluster. The size of
23 the window sets a limit to the maximum cluster that can be found,
24 So clusters can be split if their sizes exceeds the window one. We
25 show that the simplified implementation saves a large amount
26 of hardware resources and has the equivalent performance for
27 the use in the Fast TracKer processor. Finally, we describe an
28 event display that is a powerful diagnostic/monitoring tool used
29 to understand in detail the performance of the algorithm, also
30 used during the data taking.

Index Terms-Clustering, Image processing, Parallel process-

32 ing, Trigger circuits, Field programmable gate arrays, Pixel 66 33 detectors.

${ }_{40}$ reconstruction will cover the entire Silicon Detector with high ${ }_{41}$ quality events. In order to achieve these goals the first step 42 of the Fast Tracker processing needs to perform clustering of ${ }_{43}$ the Pixel detector [4] data. We focus on the most challenging 44 component on the IM card, the 2D clustering algorithm, which 45 will be executed on all pixel modules [5] data.

${ }_{46}$ The main challenge is to process the $160 \mathrm{Gbit} / \mathrm{s}$ input data. ${ }_{47}$ Data is received over $132 \mathrm{~S}$-Link fibers each running at ${ }_{48} 1.2 \mathrm{Gbits} / \mathrm{s}$. The data format consist of 32 bit words at a rate of $4940 \mathrm{MHz}$ and each word corresponds to one detector hit, with its 50 coordinates (row and column within the module) and its Time 51 over Threshold (ToT) information encoded [5]. The ability 52 to process hits at a $40 \mathrm{MHz}$ rate is required. The clustering ${ }_{53}$ algorithm must provide high quality resolution, in order to 54 match the high quality required by the HLT.

In order to achieve sufficient speed combined with the ${ }_{56}$ required level of quality, the algorithm is split in three func57 tional modules. The first module reorders the hits, which 58 initially arrive randomly, into an order appropriate for their fast 59 processing from the following modules. The second module so groups together hits belonging to the same cluster. This is ${ }_{61}$ the most time-consuming part of the algorithm where we will 62 exploit a dedicated logic in order to achieve linear processing ${ }_{63}$ time. The final module analyzes the hits of the cluster in order 64 to calculate the cluster properties of interest, such as the center 65 of mass of the cluster.

Powerful FPGAs with their logic distributed in a 2D struc${ }_{67}$ ture are perfectly suitable for mapping the 2D array of a ${ }_{68}$ pixel module. The 2D grid of clustering cells implemented ${ }_{69}$ with FPGA logic array can work in parallel to speed-up 70 substantially the first step of the algorithm. In addition the

\section{INTRODUCTION}

The algorithm implementations presented in this paper 71 FPGA offers the needed flexibility to provide results as precise 36 were developed for the Fast TracKer (FTK) [1], which is 72 as needed for the second step.

${ }_{37}$ an approved ATLAS trigger upgrade [2]. The Fast Tracker

38 will provide reconstructed tracks as input to the High Level з9 Trigger (HLT) [3], in addition to the raw detector hits. Track

\section{The ALgORithm IMPLEMENTATIONS}

74 Two different strategies have been used for the 2D clustering

This project receives support from Istituto Nazionale di Fisica Nucleare 75 inside FTK. These strategies use different approaches in order and from the European Union's Seventh Framework Marie Curie Program for 76 to form the clusters of hits on the pixel module, consisting research, technological development and demonstration under grant agreement 77 of $328 \times 144$ cells. Both strategies aim to group hits on the
n.324318.

78 pixel detector modules which are connected either side-to-side

Cisa, Largo B. Pontecorvo 3, 56127 Pisa, Italy.
A or diagonally, thus to create a cluster, and then calculate the

A.Annovi is with INFN, Sezione di Pisa, Largo Bruno Pontecorvo 3, 56127 so center of mass of the produced cluster. The centroid is then Pisa, Italy.

S. Gkaitatzis, K. Kordas are with Aristotle University of Thessaloniki, 54124 Thessaloniki, Greece.

81 sent to the subsequent subsystems of the FTK and used for 82 the track fitting. 


\section{A. The "ideal" implementation}

84 The first implementation [6] used a 2D grid of logical 85 cells representing a whole pixel module. On the first step, 86 the cluster identification algorithm groups all connected hits, 87 forming the cluster. On the second step, the center of mass 88 of the cluster is calculated as a ToT weighted average of the 89 positions of its hits. The second step is completely decoupled ${ }_{90}$ from the first one. It can be modified to calculate any cluster 91 property of interest depending on the specific application. The ${ }_{92}$ ToT information is stored in the RAM while hits are being 93 loaded on the grid. During the grid readout and cluster building ${ }_{94}$ procedure, the ToT is expected to be easily retrieved and sent 95 to the second step logic.

96 The study of the hardware size and the clock speed of this 97 implementation [4] demonstrated that the whole grid needs 98 a lot of resources to meet the required timing performance. 99 The implementation done in 2008 showed that $60 \%$ of the 100 logic in the xv5vlx330 Xilinx FPGA [6], the largest of the ${ }_{135}$ of the FTK system. FTKSim processes complete ATLAS 101 Xv5vlx group, was necessary to process data from one input ${ }_{136}$ events and creates exactly the same list of tracks that will be ${ }_{102}$ S-Link. More capacious and expensive FPGAs would have ${ }_{137}$ produced by the FTK hardware. The program serves a number 103 been required.

\section{${ }_{104}$ B. The "simplified" implementation}

105 The second implementation [7] uses a small sliding grid, ${ }^{141}$ 106 a window moving on the pixel module, in order to reduce ${ }^{142}$ 107 the hardware needs. The window is moving in the sense that ${ }^{143}$ 108 during the several passes of the cluster identification process it ${ }^{144}$ 109 is virtually placed on different positions on the pixel module ${ }^{145}$ 110 and it is filled with the hits whose coordinates fall inside ${ }^{146}$ 111 the dimensions of the window. This implementation reduces ${ }^{147}$ 112 the required logic by a factor of approximately 64 compared $^{148}$ 113 with the ideal implementation [8], for the selected moving ${ }^{149}$ 114 window size. The size of the window sets a limit to the ${ }^{150}$ 115 maximum cluster that can be found, so a cluster can be split if ${ }^{151}$ 116 either of its dimensions exceed the dimensions of the window. ${ }^{152}$ 117 In addition, a simplified center of mass is calculated as the ${ }^{153}$ 118 center of a bounding box which contains the cluster. This ${ }^{154}$ 119 implementation does not use the ToT information associated ${ }^{155}$ 120 with the hits of the cluster. These simplifications are expected ${ }^{156}$ 121 to have a small impact on the tracking resolution since most ${ }^{157}$ 122 clusters are up to two pixels wide along the $\phi$ direction and ${ }^{158}$ ${ }_{123}$ the $z$ direction $^{1}$. Figure 1 shows the size of bounding boxes ${ }^{159}$ 124 containing the identified clusters created by a top quark sample ${ }^{160}$ 125 with a high pile-up. The window size is a free parameter in ${ }^{161}$ 126 the implementation and it is chosen to have a height of $21^{162}$ 127 rows and a length of 8 columns. This is a very large window ${ }^{163}$ 128 for clean single particle clusters in the Pixel detector, which ${ }^{164}$ 129 are at maximum $3 \times 5$ large [4].

\section{III. The Algorithm Simulation and Comparison}

\section{${ }_{131}$ A. The bit-accurate $2 D$ clustering simulation}

132 As any complex architecture, the 2D clustering algorithm 133 requires a careful simulation. The simulation was developed 134 as a module for FTKSim [9], which is a complete simulation

${ }^{1} \phi$ and $z$ coordinates refer to the cylindrical geometry of pixel modules in ${ }^{172}$ the barrel detector.

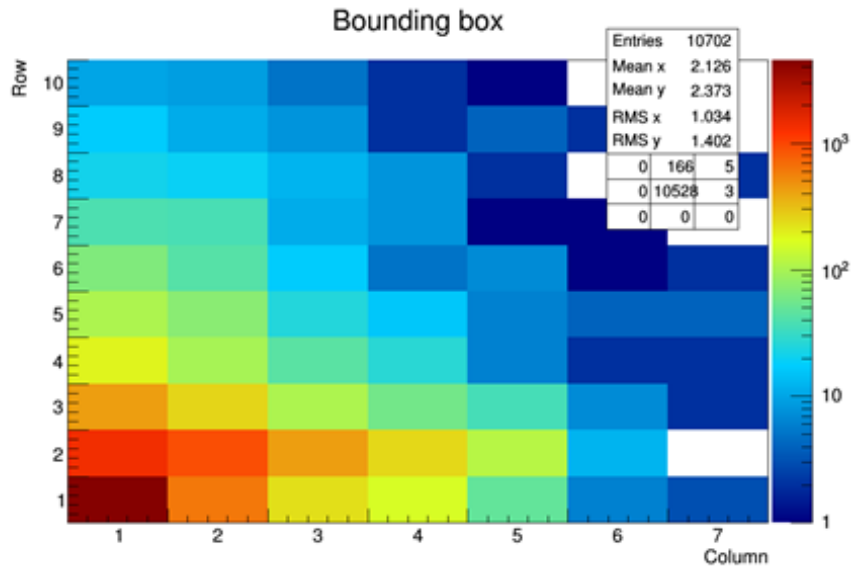

Fig. 1. Cluster dimensions for a top quark sample. 38 of purposes, such as:

- validation of the hardware,

- detailed and reliable evaluation of FTK physics performance by processing complete events produced by the full ATLAS detector simulation,

- evaluation of crucial parameters needed for the hardware design and optimization,

- determination of the large set of constants needed for programming FTK, and

- determination of tracking performance parameters for use in fast parametric detector simulation for high statistics studies of physics performance.

In the case of the clustering algorithm FTKsim is bitaccurate and is able to reproduce perfectly both the ideal and simplified implementation. The simplified hardware conditions were added:

1) the hits are ordered in the same way as the hardware to avoid any potential innacuracy in the next steps.

2) the sliding window mechanism was added in FTKsim, to search for one cluster at time. This became an option to replace the direct search of many clusters on the whole module.

3) the use of the ToT became optional and the center of mass calculation as the center of the bounding box encircling the cluster became available.

The methods used for the cluster identification and for the ${ }_{166}$ has three different values for the three different combinations 167 of the available methods: (1) PCM1 selecting the ideal method 168 for both cluster identification and centroid calculation; (2) ${ }_{169}$ PCM100 selecting the simplified clustering but the ideal cen770 troid calculation; (3) PCM101 using both simplified methods. B. Simulation results

In this section, the effect of the selected methods on the 173 resolution of the output tracks of the FTK is examined. For 
174 the comparison only one of the track parameters is shown, the 175 pseurorapidity $\eta$.

176 Two types of simulated event samples were used to compare 177 the three options. The first is a very clean sample consisting 178 of single muons without any superposition of soft collisions 179 (pile-up) which was simulated with a flat distribution in the 180 particle helix parameters. The second is an extremely complex ${ }_{181}$ sample of top quark events with an overlay of an average of ${ }_{182} 60$ pile-up events and a center of mass energy $\sqrt{s}=14 \mathrm{TeV}$. ${ }_{183}$ Figure 2 and Figure 3 show the resolution of the track ${ }_{184}$ parameter $\eta$ for the single muon and the top quark sample 185 respectively. In both figures, the shaded area corresponds 186 to PCM1, the red line to PCM100 and the blue line to ${ }_{187}$ PCM101. The switching between PCM1 and PCM100, by 188 introducing only the realistic clustering algorithm, creates a 189 minor deviation from the ideal implementation. As it can be 190 seen in the legend of Figure 1, the percentage of clusters 191 reaching the end of the window is $1.6 \%$ for the top quark 192 sample, which is very complex, meaning that the percentage of 193 split clusters will be smaller than that. However, the simplified 194 centroid calculation has a bigger effect on the resolution, with 195 the effect being more noticeable on the tails of the distribution 196 although this is partially obscured the statistics is low.

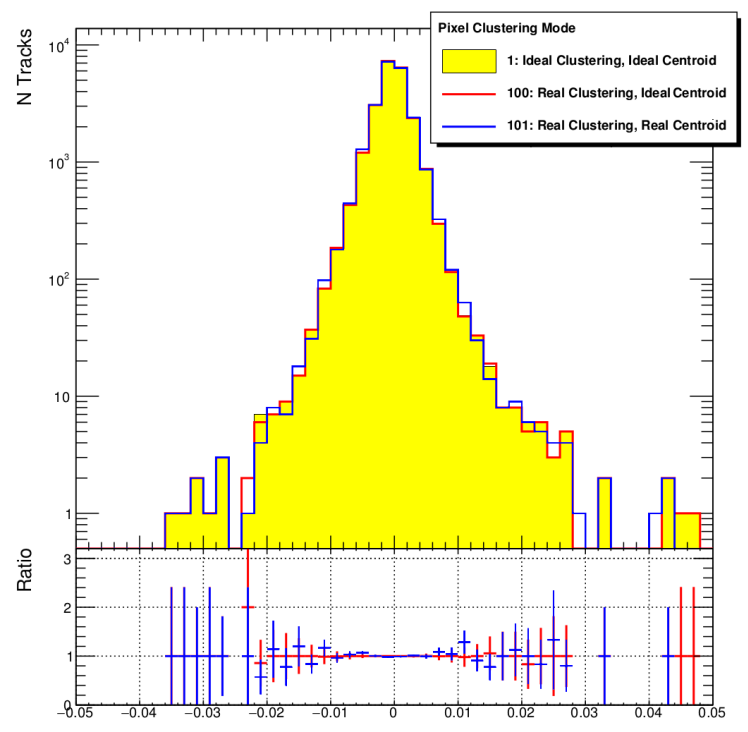

Fig. 2. Resolution of track parameter $\eta$ for the single muon sample.

197 Since the simplified implementation could potentially split a 198 cluster, and thus create a fake hit in the detector, it is important 199 to examine the impact of these extra clusters on the quality 200 of the final FTK tracks. The quality of the track fitting should 201 provide a valid indication on this effect. The FTK examines 202 the $\chi^{2}$ of the fit by performing a linear calculation [9]. The 203 calculation is a set of scalar products of the hit coordinates 204 and pre-calculated constants that take into account the detector 205 geometry and alignment. First, FTK examines the tracks and 206 keeps the ones that pass a $\chi^{2} /$ ndof cut. In case that there are 207 hits on all the detector layers but the $\chi^{2}$ fails the cut, the ${ }^{220}$ 208 track is re-fitted with one hit dropped each time. This allows 221 209 the system to recover from a random hit, which can be either 222 principle, an event display able to portray the hardware oper210 a random hit, or a split cluster. Also, FTK examines possible 223 ation has been developed in C++11 using the Qt toolkit. The

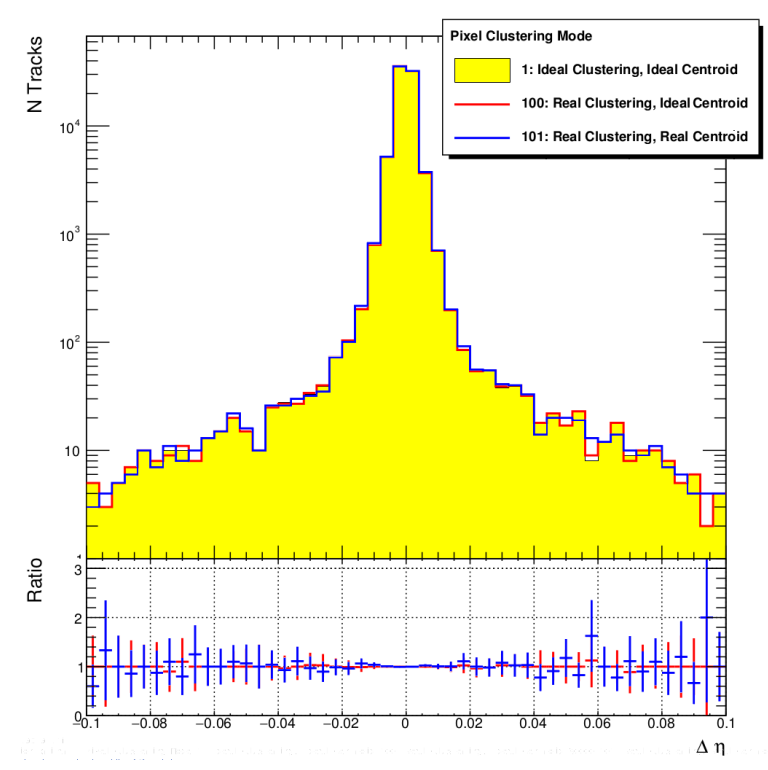

Fig. 3. Resolution of track parameter $\eta$ for the top quark sample.

211 duplicate tracks. In case two tracks are considered duplicated, ${ }_{212}$ FTK keeps the one with the best $\chi^{2}$ fit.

${ }_{213}$ Figure 4 and Figure 5 show the $\chi^{2}$ distribution for the 214 single muon and the top quark sample respectively. The green 215 line represents PCM1, the red line PCM100, while the blue 216 line represents PCM101. The ideal implementation and the 217 implementation using the simplified centroid match exactly. ${ }_{218}$ However, PCM101 leads to a loss of events, as seen on the 219 ratio plot.

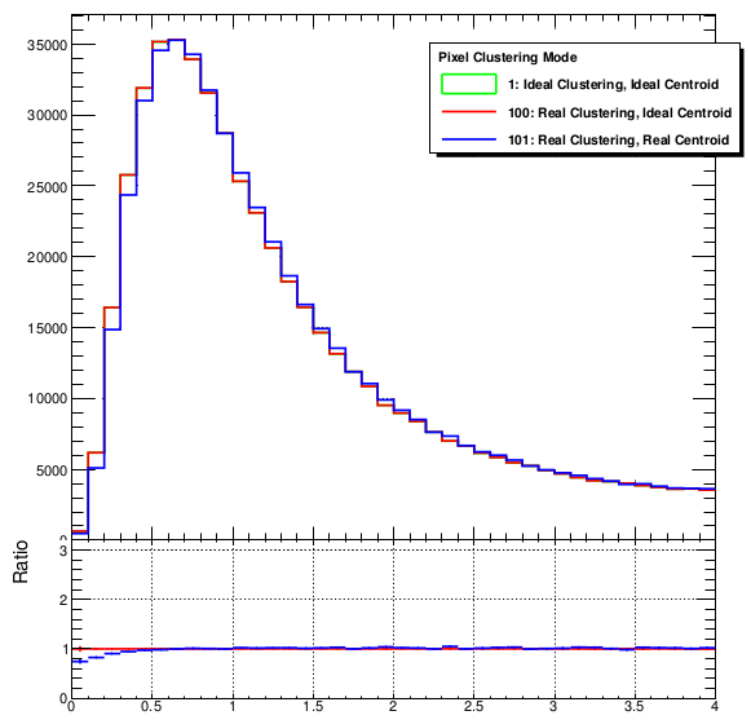

Fig. 4. $\chi^{2}$ distribution for single muon sample.

\section{DEMONSTRATOR}

In order to gain a better understanding of the operating 


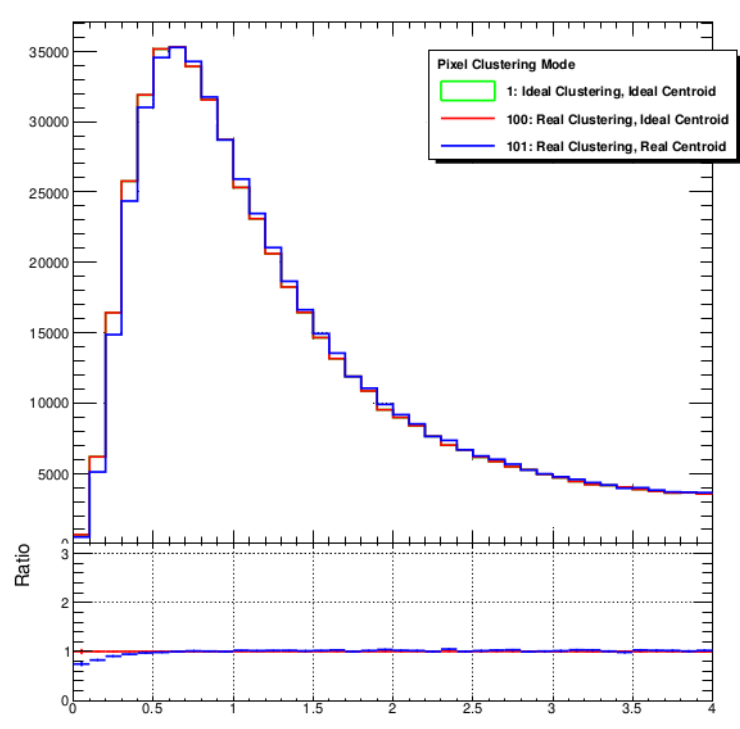

Fig. 5. $\chi^{2}$ distribution for top quark sample.

224 original implementation of the event display demonstrator $\operatorname{was}_{262}^{26}$ 225 designed as a standalone program but the design is flexible in 263 226 order to allow integration with the FTKSim. Furthermore, the ${ }_{265}^{264}$ ${ }_{227}$ modular design allows the user to choose freely any combi- ${ }_{265}^{265}$ 228 nation of clustering implementations and centroid calculation $267[4]$ 229 modes. Therefore, it can provide valuable insight for the future 268 230 development of the firmware, allowing a visualization of the ${ }_{270}^{269}$ ${ }_{231}$ centroid location.

232 The display can accept the same input as the hardware ${ }_{273}^{272}$ 233 and then perform an accurate simulation of the hardware.274 ${ }_{234}$ The results are presented on a $2 \mathrm{D}$ grid which represents $\mathrm{a}^{275}$ ${ }_{235}$ pixel or an Insertable B-Layer (IBL) module. Alternatively, ${ }_{277}^{276}$ 236 the display is able to receive data from the hardware via spy 278 ${ }_{237}$ buffers. Figure 6 shows a small active region of a pixel module ${ }^{279}$ 238 taken from the event display. Each cell is color-coded based on ${ }_{281}^{280}$ 239 the charge deposited on the pixel, with blue representing the ${ }_{282}$ 240 lowest ToT and red the highest. The centroid is represented ${ }^{283}$ 241 by a magenta circle. The moving window is represented by ${ }_{285}^{284}$ 242 the yellow region. The window on the demonstrator follows 243 the same movement as the window on the hardware. In this 244 example, it can be seen that the largest cluster has been split 245 (two magenta centroids appear on it) since it exceeded the 246 width of the window ( 8 columns $\times 21$ rows). Consequently, 247 modifications of the algorithms can be evaluated and under248 stood directly.

\section{CONCLUSIONS}

250 Two different implementations of a 2D clustering algorithm 251 for pixel detector data have been compared to evaluate their 252 relative performance. The simplified implementation, which 253 reduces the required logic by a factor of approximately 64 for 254 the selected window size, has the equivalent performance as 255 the ideal implementation when they are compared using two 256 event samples simulated with the FTK system.

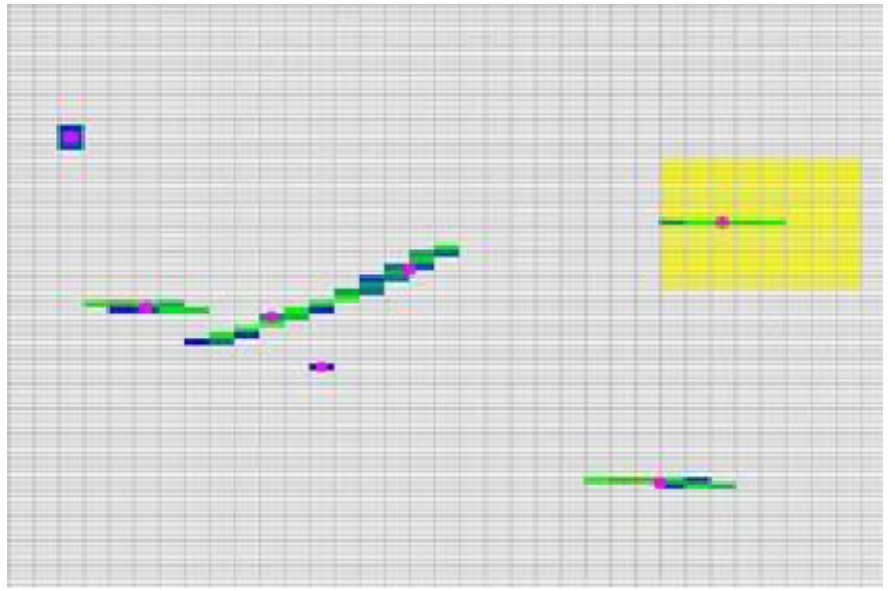

Fig. 6. Region of a pixel module created with the demonstrator.

] ATLAS Collaboration, "The ATLAS Experiment at the CERN Large Hadron Collider," Journal of Instrumentation, vol. 3, no. 08, pp. S08 003-S08 003, Aug. 2008.

3] ATLAS Collaboration, "ATLAS high-level trigger, data acquisition and controls: Technical design report," CERN-LHCC-2003-022, ATLAS-TRD016, 2003.

4] ATLAS Collaboration, ATLAS pixel detector: Technical Design Report, ser. Technical Design Report ATLAS. Geneva: CERN, 1998.

[5] G. Aad et al., "ATLAS pixel detector electronics and sensors," Journal of Instrumentation, vol. 3, no. 07, pp. P07 007-P07 007, Jul. 2008.

[6] Annovi, A. and Beretta, M., "A fast general-purpose clustering algorithm based on FPGAs for high-throughput data processing," Nuclear Instruments and Methods in Physics Research Section A: Accelerators, Spectrometers, Detectors and Associated Equipment, vol. 617, no. 1-3, pp. 254-257, May 2010.

7] C.-L. Sotiropoulou, A. Annovi, M. Beretta, P. Luciano, S. Nikolaidis, and G. Volpi, "A multi-core FPGA-based clustering algorithm for real-time image processing." IEEE, Oct. 2013, pp. 1-5.

[8] C.-L. Sotiropoulou, S. Gkaitatzis, A. Annovi, M. Beretta, P. Giannetti, K. Kordas, P. Luciano, S. Nikolaidis, C. Petridou, and G. Volpi, "A MultiCore FPGA-Based 2d-Clustering Implementation for Real-Time Image Processing," IEEE Transactions on Nuclear Science, vol. 61, no. 6, pp. 3599-3606, Dec. 2014.

[9] ATLAS Collaboration, "Fast TracKer (FTK) Technical Design Report," Jun. 2013. 